\title{
Tumor-preventing activity of aspirin in multiple cancers based on bioinformatic analyses
} \author{
Zheng He ${ }^{4}$, Meiling Jin ${ }^{\text {Corresp., }} 5$, Changting Liu Corresp. 1 \\ ${ }^{1}$ Chinese PLA General Hospital, Nanlou Respiratory Diseases Department, Beijing, China \\ 2 Chinese PLA General Hospital, Nanlou Medical Oncology Department, Beijing, China \\ 3 Hebei Medical University, School of Chinese Integrative Medicine, Shijiazhuang, China \\ 4 Chinese PLA General Hospital, Department of Clinical Laboratory, Beijing, China \\ 5 Beijing Chao-yang Hospital, Department of Nephrology, Beijing, China \\ Corresponding Authors: Meiling Jin, Changting Liu \\ Email address: auml_1986@hotmail.com, changtingliu1212@sohu.com
}

Diangeng Li ${ }^{1}$, Peng Wang ${ }^{2}$, Yi Yu ${ }^{1}$, Bing Huang ${ }^{1}$, Xuelin Zhang ${ }^{1}$, Chou Xu ${ }^{1}$, Xian Zhao ${ }^{1}$, Zhiwei Yin ${ }^{3}$,

Background: Acetylsalicylic acid was renamed aspirin in 1899, and it has been widely used for its multiple biological actions. Because of the diversity of the cellular processes and diseases that aspirin reportedly affects and benefits, uncertainty remains regarding its mechanism in different biological systems. Methods: The Drugbank and STITCH databases were used to find direct protein targets (DPTs) of aspirin. The Mentha database was used to analyze protein-protein interactions (PPIs) to find DPT-associated genes. DAVID was used for the GO and KEGG enrichment analyses. The cBio Cancer Genomics Portal database was used to mine genetic alterations and networks of aspirin-associated genes in cancer. Results: Eighteen direct protein targets (DPT) and 961 DPT-associated genes were identified for aspirin. This enrichment analysis resulted in eight identified KEGG pathways that were associated with cancers. Analysis using the cBio portal indicated that aspirin might have effects on multiple tumor suppressors, such as TP53, PTEN, and RB1 and that TP53 might play a central role in aspirin-associated genes. Discussion: The results not only suggest that aspirin might have anti-tumor actions against multiple cancers but could also provide new directions for further research on aspirin using a bioinformatics analysis approach. 


\section{Tumor-preventing activity of aspirin in multiple cancers based on}

\section{bioinformatic analyses}

3 Diangeng $\mathrm{Li}^{1 *}$, Peng Wang ${ }^{3 *}$, Yi Yu ${ }^{1}$, Bing Huang ${ }^{1}$, Xuelin Zhang ${ }^{1}$, Chou Xu ${ }^{1}$, Xian Zhao ${ }^{1}$, Zhiwei Yin ${ }^{4}$,

$5 \quad{ }^{1}$ Nanlou Respiratory Diseases Department, Chinese PLA General Hospital, Chinese PLA Psotgraduate

6 Medical School, Beijing, 100853, China

$7 \quad{ }^{2}$ Department of Nephrology, Beijing Chao-yang Hospital, Beijing, 100020, China

$8 \quad{ }^{3}$ Nanlou Medical Oncology Department, Chinese PLA General Hospital, Beijing, 100853, China

$9{ }^{4}$ School of Chinese Integrative Medicine, Hebei Medical University, Shijiazhuang 050017, China

$10{ }^{5}$ Department of Clinical Laboratory, Chinese PLA General Hospital, Beijing, 100853, China

$11{ }^{*}$ Equal contributors

$12{ }^{*}$ Co-Corresponding author

\section{Correspondence:}

14 Changting Liu, MD, PhD,

15 Nanlou Respiratory Diseases Department, Chinese PLA General Hospital, Chinese PLA Psotgraduate Medical

16 School, Beijing, 100853, China

17 E-mail: changtingliu1212@sohu.com

18 Telephone: 010-66876432

Fax: 010-66876432 


\section{Abstract}

24 Background: Acetylsalicylic acid was renamed aspirin in 1899, and it has been widely used for its multiple biological actions. Because of the diversity of the cellular processes and diseases that aspirin reportedly affects and benefits, uncertainty remains regarding its mechanism in different biological systems.

Methods: The Drugbank and STITCH databases were used to find direct protein targets (DPTs) of aspirin. The Mentha database was used to analyze protein-protein interactions (PPIs) to find DPT-associated genes. DAVID was used for the GO and KEGG enrichment analyses. The cBio Cancer Genomics Portal database was used to mine genetic alterations and networks of aspirinassociated genes in cancer.

Results: Eighteen direct protein targets (DPT) and 961 DPT-associated genes were identified for aspirin. This enrichment analysis resulted in eight identified KEGG pathways that were associated with cancers. Analysis using the cBio portal indicated that aspirin might have effects on multiple tumor suppressors, such as TP53, PTEN, and RB1 and that TP53 might play a central role in aspirin-associated genes.

Discussion: The results not only suggest that aspirin might have anti-tumor actions against multiple cancers but could also provide new directions for further research on aspirin using a bioinformatics analysis approach. 
42

43

44

45

46

47

\section{Introduction}

Nonsteroidal anti-inflammatory drugs (NSAIDs) are efficacious preventive agents against several different types of malignancies, including colorectal cancer (Bilani et al. 2017). Reports regarding risk reduction have shown impressive results with increasing NSAID intake showing a reduced relative risk of colon cancer by $63 \%$, whereas it has shown a $39 \%$ reduction for prostate and breast cancer and 36\% for lung cancer (Harris et al. 2005). A long-term observation of randomized, controlled trial cohorts with cardiovascular disease also revealed lower risks of developing colon malignancy and a reduced incidence and development of metastatic disease, which are benefits that are attributed to regular aspirin use (Gray et al. 2017).

The recent advancements in biomedical research, such as multicenter genomic studies involving proteomics, microarrays and other high-throughput screening assays, has resulted in a staggering amount of candidate gene "hits"; more than enough to overwhelm subsequent thematic or phenotypic-based data analyses. Nevertheless, the network-based approach can be a simple and effective means of analyzing these gargantuan sets of data and permit researchers to uncover previously difficult to characterize genetic relationships between a drug, its targets and interacting proteins as well as its disease associations. It has been reported that establishing a drug target network can be accomplished using drug interaction databases (Mestres et al. 2008). There are several open-access databases for the collection of pharmacogenomics data. Drugbank is the most commonly used database. Drugbank's primary focus is compiling and curating information concerning drug targets (genetic and protein-specific data), drug metabolism, drug interactions, and the relationships between drugs and diseases or side effects (Wishart 2008). 
63 However, Drugbank might not completely overlap with those in STITCH or the Therapeutic

64 Target Database. In this study, we first identified direct protein targets (DPTs) using Drugbank

65 and the STITCH database. We then identified proteins associated with these DPTs using the

66 Mentha database. Finally, we built an aspirin-target network. Enrichment analysis was used to

67 analyze the proteins of this network. This method of analysis permits a deeper understanding of

68 how aspirin may prevent cancer and drive the development of future chemotherapeutic

69 medication.

\section{Materials and Methods}

\section{Drug-target search}

In this study, Drugbank (https://www.drugbank.ca/) (Wishart et al. 2006) and STITCH

(http://stitch.embl.de/) (Kuhn et al. 2007) were utilized to identify aspirin-target interactions to

produce an aspirin-target network. A visualization chart was constructed with the resultant data,

followed by more extensive data analysis and proposals for subsequent validation experiments.

to find DPT-associated genes with the 0.3 set as the minimum interaction scores (Calderone et al. 2013). DAVID was used for the GO enrichment analysis and KEGG enrichment analysis (Huang et al. 2008).

Aspirin-linked cancer genomic data exploration using the cBio Cancer Genomics Portal 
84 allows multidimensional exploration of cancer genomic data by translating molecular profiles sequenced from cell lines and cancer tissues into easily comprehensible proteomic, gene expression, epigenetic and genetic events (Cerami et al. 2012). With the cBio Portal, we explored the connections of aspirin-associated genes across the genetic databases of several cancer-related studies. Using the portal search function, all of the aspirin-associated genes found in cancer study samples were categorized as altered or not altered. We were also able to construct multiple visualization platforms by grouping the cancer data alterations based on aspirin gene data sets.

\section{Results}

\section{Identification of DPTs}

Drugbank and STITCH were used to identify direct protein targets (DPTs) of aspirin; these 18 primary DPTs of aspirin were PTGS1, PTGS2, AKR1C1, PRKAA1, EDNRA, IKBKB, TP53, HSPA5, RPS6KA3, NFKBIA, NFKB2, CRP, SELP, TBXA2R, REN, MMP9, NOS3, and IL10. Then, we used Mentha to analyze protein-protein interactions (PPIs) to find DPT-associated genes and uncovered 961 unique target-protein interactions, which we determined to be aspirinrelated DPT-associated genes along with the 18 primary targets (Table 1).

\section{GO pathway analysis}

The online DAVID software was used to determine overrepresented GO categories based on our previously identified DPT-associated genes. GO analysis revealed significant genetic enrichment in the area of biological processes (BP), which was comprised of the regulation of nucleobase, nucleoside, nucleotide and nucleic acid metabolism (27.4\%), signal transduction 
105 (27.2\%), cell communication (23.4\%), protein metabolism (16.7\%), apoptosis (3.9\%), regulation

106 of gene expression and epigenetics (1.5\%), regulation of the cell cycle $(1.5 \%)$, DNA repair

$107(1.4 \%)$, regulation of cell growth $(0.8 \%)$, and regulation of cell proliferation $(0.8 \%)$. For the area

108 of the cell components (CC), these genes were enriched in the nucleus (70.6\%), cytoplasm

109 (64.3\%), nucleolus (21.5\%), cytosol (21\%), exosomes (20.1\%), nucleoplasm (13.2\%),

110 centrosome $(11.8 \%)$, ribonucleoprotein complex $(2.4 \%)$, protein complex $(2.1 \%)$ and PML body

$111(1.9 \%)$. Additionally, GO molecular function (MF) analyses showed that these genes were

112 significantly enriched in transcription regulator activity (11.3\%), ubiquitin-specific protease

113 activity (9.9\%), transcription factor activity (9.7\%), protein serine/threonine kinase activity

$114(8.7 \%)$, receptor signaling complex scaffold activity $(5.3 \%)$, chaperone activity $(2.5 \%)$, protein

115 binding $(2.3 \%)$, protein serine/threonine phosphatase activity (1.1\%), DNA repair protein

$116(1.1 \%)$, and DNA topoisomerase activity (0.4\%) (Figure 1).

\section{KEGG pathway analysis}

118 The functional characteristics of these aspirin-related genes were characterized by the use of

119 the KEGG pathway enrichment analysis, which is a feature embedded in the DAVID software.

120 The top 10 KEGG pathways linked to aspirin DPTs and their DPT-associated genes include

121 Epstein-Barr virus infection (63 genes), ubiquitin-mediated proteolysis (46 genes), pathways in

122 cancer (78 genes), cell cycle (40 genes), NF-kappaB signaling pathway (33 genes), herpes

123 simplex infection (47 genes), TNF signaling pathway (35 genes), toxoplasmosis (37 genes), viral

124 carcinogenesis (52 genes), and FOXO signaling pathway (37 genes). We primarily focused on

125 the KEGG pathways associated with cancers: prostate cancer (28 genes), pancreatic cancer (21 
126

127

128

129

130

131

132

133

134

135

136

137

138

139

140

141

142

143

144

145

146

genes), small-cell lung cancer (24 genes), colorectal cancer (16 genes), bladder cancer (11

genes), endometrial cancer (11 genes), non-small-cell lung cancer (11 genes), and renal cell

carcinoma (11 genes; Table 2).

Mining genetic alterations and networks of aspirin-associated genes in cancer with the cBio

portal

Prostate cancer

There were large variations of $24.23 \%$ to $73.3 \%$ in the gene sets analyzed among 9 prostate cancer gene analysis studies. OncoPrint results showed that $1412(50 \%)$ cases had an alteration in at least one of these 28 gene sets (PTEN 18\%, TP53 16\%, RB1 8\%, IKBKB 7\%, HDAC2 7\%, FGFR1 6\%, PIK3R1 5\%) (Figure 2A and Supplemental Figure 1). With the help of the CBio portal, we were able to obtain interactive analyses and view constructed networks of genes that were altered in cancer. Figure 3A depicts a gene network consisting of PTEN, TP53, and IKBKB genes and their respective gene neighbors. PTEN and TP53 may play important roles in this network.

\section{Pancreatic cancer}

The gene sets for the five analyzed pancreatic cancer studies revealed variations of $35.35 \%$ to $87.16 \%$ among the gene sets. The results showed that $670(70 \%)$ cases had an alteration in at least one of these gene sets (TP53 58\%, SMAD2 4\%, TGFB1 2.5\%, AKT1 2.3\%, RB1 1.9\%,

SMAD3 1.7\%, and STAT3 1.7\%) (Figure 2B and Supplemental Figure 2). As shown in Figure

3B, TP53 may play an important role in this network.

\section{Small-cell lung cancer}


Upon the analysis of 4 small-cell lung cancer studies, we noted alterations of $78.43 \%$ to

$148100 \%$ between the gene sets. The OncoPrint results showed that $193(91.9 \%)$ cases had an

149 alteration in at least one of the 24 gene sets (TP53 86\%, RB1 65\%, FN1 12\%, PTEN 8\%,

150 LAMC3 5\%, NOS2 4\%, and LAMA4 3\%) (Figure 2C and Supplemental Figure 3). As shown in

151 Figure 3C, there was a close relationship between TP53 and RB1, and TP53 may play an important role in this network.

153

\section{Colorectal cancer}

There were variations of $31.41 \%$ to $84.78 \%$ for the five colorectal cancer study gene sets that we interpreted. The results showed that $892(51.1 \%)$ cases had an alteration in at least one of these gene sets (TP53 37\%, SMAD2 5\%, CTNNB1 4\%, PIK3R1 4\%, and SMAD3 3\%) (Figure 2D and Supplemental Figure 4). We focused primarily on TP53, and the network of TP53 is shown in Figure 3D.

\section{Bladder cancer}

We observed alterations ranging from $36.08 \%$ to $91.18 \%$ in gene sets from the 9 analyzed bladder cancer studies. The results show that $1316(74.9 \%)$ cases had an alteration in at least one of the 11 gene sets (TP53 41\%, CDKN2A 31\%, RB1 20\%, CDKN1A 9\%, and MDM2 8\%)

(Figure 2E and Supplemental Figure 5). The network of these genes is shown in Figure 3E, and TP53 and CDKN2A may play an important role in this network.

\section{Endometrial cancer}

The three endometrial genetic studies that we analyzed had gene set variations ranging from $49.6 \%$ to $94.33 \%$. The results showed that $1036(71 \%)$ cases had an alteration in at least one of 
168

169

170

171

172

173

174

175

176

177

178

179

180

181

182

183

184

185

186

187

these gene sets (PTEN 48\%, PIK3R1 24\%, TP53 23\%, CTNNB1 20\%, and AXIN1 5\%) (Figure

2F and Supplemental Figure 6). The network of these genes is shown in Figure 3F.

Non-small-cell lung cancer

Among the analyzed NSCL cancer studies, alterations ranging from $40.61 \%$ to $97.19 \%$

were found for the submitted gene sets. The results showed that 2046 (64\%) cases had an

alteration in at least one of these gene sets (TP53 49\%, CDKN2A 25\%, EGFR 17\%, PIK3CA

9\%, and RB1 7\%) (Figure 2G and Supplemental Figure 7). The network of these genes is shown

in Figure 3G. This indicates that TP53 may play important roles in the occurrence of NSCLC.

\section{Renal cell carcinoma}

The renal cell carcinoma studies included in our analysis displayed intergene set alterations of $4.11 \%$ to $78.48 \%$. The results showed that $827(30 \%)$ cases had an alteration in at least one of these gene sets (VHL 27\%, CREBBP 1.4\%, and AKT1 0.6\%) (Figure 2H and Supplemental Figure 8). The network of these genes is shown in Figure 3H.

\section{Discussion}

Acetylsalicylic acid was renamed aspirin in 1899 (Fuster \& Sweeny 2011). In 1988, a casecontrol study was the first to record a negative correlation between colorectal cancer and aspirin use (Kume et al. 2010), which suggests that aspirin might be protective against cancer. Further investigations based on cohorts of cardiovascular disease patients taking aspirin found that aspirin may generally lower the risk of cancer. Six separate trials that analyzed patients who took daily low-dose aspirin ( $75 \mathrm{mg}$ and above) for three years revealed that aspirin conferred an 
overall relative risk of 0.76 for cancer with a longer duration of aspirin intake resulting in higher benefits (Rothwell et al. 2012). In fact, several lines of evidence highlight that aspirin may be beneficial in decreasing mortality in cancer, especially colorectal cancer-related death. This protection may also extend to other malignancies, such as prostate, lung, breast and gastroesophageal cancers. Given the strong epidemiological evidence, it is hypothesized that aspirin may act on common cancer pathways to suppress cancer progression and metastases (Cao et al. 2016). In 2007, the United States Preventive Services Task Force (USPSTF) initially discouraged aspirin use for preventing colorectal cancer. However, the updated USPSTF 2015 recommendations acknowledge the existence of several compelling sources of evidence and included colorectal cancer prevention into the rationale for routine, low-dose aspirin intake for those with specific cardiovascular risk profiles between the ages of 50 to 69 . This landmark decision was the first to endorse a pharmacological compound for use as a preventive agent against cancer in a population not specifically known to have a high risk of developing malignancies. Despite these advancements, we still possess a limited understanding of how aspirin exerts its benefits. Our study utilized bioinformatics methods to establish a drug target network to dissect the underlying molecular mechanisms of aspirin in cancer. We first determined primary aspirin DPTs and functionally linked them to their respective proteins with the help of drug interaction databases and protein-protein interaction database (Drugbank, STITCH, and Mentha). Next, using samples from large-scale cancer genomic projects in the cBio portal, we verified if there were previously identified genetic alterations that were characterized for aspirin-associated genes/proteins. This method allowed us to clearly map out 
210

211

212

213

214

215

216

217

218

219

220

221

222

223

224

225

226

227

228

229

230

aspirin-related DPTs and their associated genes to their biological pathways using the available databases. Not only does this information contribute to the current knowledge of how aspirin prevents cancer, it also uncovers potential treatment targets and provides new directions for cancer therapeutics.

Using the tools available on the online platform, we identified 18 primary DPTs, 961 secondary DPT-associated genes/proteins, and eight enriched KEGG pathways linked to aspirinassociated genes. These eight enriched KEGG pathways included several cancers. The cBio portal was used to analyze associations between these genes and cancer based on the TCGA database. The results show that most of the gene protein targets could be found to have alteration in cancer samples, and the network analysis showed that TP53, PTEN, and RB1 might play important roles in the mechanism of aspirin. Human cancers commonly display mutated or inactivated versions of the TP53 and PTEN tumor suppressor genes. TP53 is a crucial cell cycle regulator and is responsible for inducing apoptosis. As shown in Figure 3, TP53 was found to possess a central role in the gene networks that we constructed. A large proportion of genetic defects in prostate cancer were identified to be mutations or deletions that result in attenuations of TP53 and PTEN expressions and culminate in enhanced carcinogenesis. By controlling PTEN transcription, p53 can suppress tumorigenesis when there is PTEN deficiency. It has been reported that copy number alterations of $\mathrm{p} 53$ and RB1 could be prognostic markers in prostate cancer as RB1 and TP53 were found to cooperate in suppressing metastasis (Ku et al. 2017). Functionally inactivating RB1 and TP53 appeared to be enough to stimulate SCLC development in mice, whereas restoring their expression in human SCLC cell lines halted further 
231

tumorigenesis by the induction of G1-arrest and cell apoptosis (Fiorentino et al. 2016). It has been reported that mutations in TP53 and CKDN2A define the genetic landscape of pancreatic ductal adenocarcinoma. Alterations in TP53 can promote invasion and metastasis by increasing PDGFRB transcription and reversing the repressive function of the p73/NF-Y complex (Weissmueller et al. 2014). The p16 protein is encoded by the CDKN2A gene that resides on chromosome 9p21 and operates as a tumor suppressing gene. It represents a crucial cyclininhibiting cell cycle mediator, which serves to protect against premature cell transition from the G1 into the S phase. It was reported that a higher proportion of mutations occurred in CDKN2A in sample probands with familial pancreatic cancer (Zhen et al. 2015). NF- $\kappa B$ is upregulated in prostate cancer, whereas the knockdown of NF- $\mathrm{kB}$ decreased the expression of survivin, which is an important anti-apoptotic protein and NF- $\mathrm{kB}$ target gene, and induced capase-3 cleavage (Zhuang et al. 2014). Thus, IKBKB was named after its function of phosphorylating IKB molecules, which is the inhibitor of NF-kB transcription factors (Schmid \& Birbach 2008), and indicates that IKBKB could act as a tumor suppressor. The Forkhead Box $\mathrm{O}$ family of transcription factors is comprised of three principal members, FOXO1, FOXO3, and FOXO4, which facilitate intracellular processes, such as glucose metabolism, cell differentiation, cell cycle regulation and other cellular functions. As a tumor suppressor, FOXO1 negatively regulates the highly oncogenic phosphatidylinositol 3-kinase (P13K)/AKT signaling pathway (Wallis et al. 2015). For colorectal cancer, aspirin has been recommended for use in the prevention of CRC. The PIK3CA mutation has been found to be a potential predictive biomarker for CRC (Ogino et al. 2014). Among the significantly enriched pathways from the KEGG 
252 analysis, many pathways have been proven to be involved in cancer metastasis, such as the FoxO 253 signaling pathway (Lin et al. 2015), the AMPK signaling pathway (Goodwin et al. 2014), and the 254 MAPK signaling pathway (Li et al. 2016). This evidence suggests that aspirin might also take part in the process of cancer metastasis and this should be verified in the further research. It is

256 257

noteworthy that apart from the cancers identified in this study, aspirin might also have chemoprotective activity on other cancers, such as melanoma and ovarian cancer. Previous studies suggest that long-term aspirin use may be associated with a reduced risk of melanoma, especially among women (Famenini \& Young 2014; Gamba et al. 2013). Aspirin use was also associated with a reduced risk of ovarian cancer, especially among daily users of low-dose aspirin (Trabert et al. 2014). If there was a continuous annotation update in the database, then more targets would be found in aspirin.

Thus, aspirin has anti-tumorigenic and chemopreventative activities in multiple tumors based on evidence from the bioinformatics analysis. In this study, the bioinformatics analysis helped visualize the molecular network bridging connectivity between aspirin-associated genes, aspirin and its primary targets, which demonstrates that these components are functionally related. This phenomenon may be biologically linked to the clinical impact that aspirin has on cancers, which may facilitate understanding of the tumor-preventing mechanism(s) of aspirin. Then, the molecular pathological epidemiology (MPE) could be used to study the "hot" proteins/genes as biomarker and individualized treatment as well as the outcomes. Although several limitations exist in this study, such as the verification of aspirin PPI, the evidence of a drug enrichment analysis baseline, and a lack of verification of clinical outcomes, all of these 
273 limitations will be the focus of further research. By establishing an aspirin target network,

274 examining phenotypic variations in the context of aspirin-associated genes, and by characterizing

275 cancer-specific gene signatures we gained insight into the role of aspirin in the prevention and

276 treatment of diseases, including cancers.

277

278 Conclusions

279 This bioinformatics analysis approach may significantly advance drug-disease research and

280 increase our knowledge of the pathophysiology of malignant disease, which will significantly

281 enhance our ability to devise techniques that can diagnose cancer earlier and more accurately.

282 Given the rapid growth spurt in the field of aspirin biology, we hope that the results of this study

283 will be able to provide new research directions for aspirin in cancer and for other human

284 diseases.

285

286

Reference

287

288

Bilani N, Bahmad H, and Abou-Kheir W. 2017. Prostate Cancer and Aspirin Use: Synopsis of the Proposed Molecular Mechanisms. Frontiers in pharmacology 8:145. 10.3389/fphar.2017.00145

Calderone A, Castagnoli L, and Cesareni G. 2013. Mentha: a resource for browsing integrated protein-interaction networks. Nature methods 10:690.

Cao Y, Nishihara R, Wu K, Wang M, Ogino S, Willett WC, Spiegelman D, Fuchs CS, Giovannucci EL, and Chan AT. 2016. Population-wide Impact of Long-term Use of Aspirin and the 
295

296

297

298

299

300

301

302

303

304

305

306

307

308

309

310

311

312

313

314

Cerami E, Gao J, Dogrusoz U, Gross BE, Sumer SO, Aksoy BA, Jacobsen A, Byrne CJ, Heuer ML, and Larsson E. 2012. The cBio cancer genomics portal: an open platform for exploring multidimensional cancer genomics data. AACR.

Famenini S, and Young LC. 2014. Aspirin use and melanoma risk: a review of the literature. Journal of the American Academy of Dermatology 70:187-191.

Fiorentino FP, Tokgun E, Sole-Sanchez S, Giampaolo S, Tokgun O, Jauset T, Kohno T, Perucho M, Soucek L, and Yokota J. 2016. Growth suppression by MYC inhibition in small cell lung cancer cells with TP53 and RB1 inactivation. Oncotarget 7:31014-31028. 10.18632 /oncotarget. 8826

Fuster V, and Sweeny JM. 2011. Aspirin: a historical and contemporary therapeutic overview. Circulation 123:768-778. 10.1161/CIRCULATIONAHA.110.963843

Gamba CA, Swetter SM, Stefanick ML, Kubo J, Desai M, Spaunhurst KM, Sinha AA, Asgari MM, Sturgeon S, and Tang JY. 2013. Aspirin is associated with lower melanoma risk among postmenopausal Caucasian women: the Women's Health Initiative. Cancer 119:1562-1569.

Goodwin JM, Svensson RU, Lou HJ, Winslow MM, Turk BE, and Shaw RJ. 2014. An AMPKindependent signaling pathway downstream of the LKB1 tumor suppressor controls Snail1 and metastatic potential. Molecular cell 55:436-450.

Gray RT, Cantwell MM, Coleman HG, Loughrey MB, Bankhead P, McQuaid S, O'Neill RF, Arthur K, Bingham V, McGready C, Gavin AT, Cardwell CR, Johnston BT, James JA, 
315

316

317

318

319

320

321

322

323

324

325

326

327

328

329

330

331

332

333

334

335

Hamilton PW, Salto-Tellez M, and Murray LJ. 2017. Evaluation of PTGS2 Expression, PIK3CA Mutation, Aspirin Use and Colon Cancer Survival in a Population-Based Cohort Study. Clinical and translational gastroenterology 8:e91. 10.1038/ctg.2017.18

Harris RE, Beebe-Donk J, Doss H, and Burr Doss D. 2005. Aspirin, ibuprofen, and other nonsteroidal anti-inflammatory drugs in cancer prevention: a critical review of non-selective COX-2 blockade (review). Oncology reports 13:559-583.

Huang DW, Sherman BT, and Lempicki RA. 2008. Systematic and integrative analysis of large gene lists using DAVID bioinformatics resources. Nature protocols 4:44.

Ku SY, Rosario S, Wang Y, Mu P, Seshadri M, Goodrich ZW, Goodrich MM, Labbe DP, Gomez EC, Wang J, Long HW, Xu B, Brown M, Loda M, Sawyers CL, Ellis L, and Goodrich DW. 2017. Rb1 and Trp53 cooperate to suppress prostate cancer lineage plasticity, metastasis, and antiandrogen resistance. Science 355:78-83. 10.1126/science.aah4199

Kuhn M, von Mering C, Campillos M, Jensen LJ, and Bork P. 2007. STITCH: interaction networks of chemicals and proteins. Nucleic acids research 36:D684-D688.

Kume S, Uzu T, Horiike K, Chin-Kanasaki M, Isshiki K, Araki S, Sugimoto T, Haneda M, Kashiwagi A, and Koya D. 2010. Calorie restriction enhances cell adaptation to hypoxia through Sirt1-dependent mitochondrial autophagy in mouse aged kidney. The Journal of clinical investigation 120:1043-1055. 10.1172/JCI41376

Li P, Xue W-J, Feng Y, and Mao Q-S. 2016. Long non-coding RNA CASC2 suppresses the proliferation of gastric cancer cells by regulating the MAPK signaling pathway. American journal of translational research 8:3522. 
336

337

338

339

340

341

342

343

344

345

346

347

348

349

350

351

352

353

354

355

356

Lin C-H, Chang C-Y, Lee K-R, Lin H-J, Chen T-H, and Wan L. 2015. Flavones inhibit breast cancer proliferation through the Akt/FOXO3a signaling pathway. BMC cancer 15:958.

Mestres J, Gregori-Puigjane E, Valverde S, and Sole RV. 2008. Data completeness - the Achilles heel of drug-target networks. Nature biotechnology 26:983.

Ogino S, Lochhead P, Giovannucci E, Meyerhardt JA, Fuchs CS, and Chan AT. 2014. Discovery of colorectal cancer PIK3CA mutation as potential predictive biomarker: power and promise of molecular pathological epidemiology. Oncogene 33:2949-2955. 10.1038/onc.2013.244

Rothwell PM, Price JF, Fowkes FG, Zanchetti A, Roncaglioni MC, Tognoni G, Lee R, Belch JF, Wilson M, Mehta Z, and Meade TW. 2012. Short-term effects of daily aspirin on cancer incidence, mortality, and non-vascular death: analysis of the time course of risks and benefits in 51 randomised controlled trials. Lancet 379:1602-1612. 10.1016/S01406736(11)61720-0

Schmid JA, and Birbach A. 2008. IkappaB kinase beta (IKKbeta/IKK2/IKBKB)--a key molecule in signaling to the transcription factor NF-kappaB. Cytokine \& growth factor reviews 19:157-165. 10.1016/j.cytogfr.2008.01.006

Trabert B, Ness RB, Lo-Ciganic W-H, Murphy MA, Goode EL, Poole EM, Brinton LA, Webb PM, Nagle CM, and Jordan SJ. 2014. Aspirin, nonaspirin nonsteroidal anti-inflammatory drug, and acetaminophen use and risk of invasive epithelial ovarian cancer: a pooled analysis in the Ovarian Cancer Association Consortium. JNCI: Journal of the National Cancer Institute 106. 
357 Wallis CJ, Gordanpour A, Bendavid JS, Sugar L, Nam RK, and Seth A. 2015. MiR-182 Is

358 Associated with Growth, Migration and Invasion in Prostate Cancer via Suppression of

359

360

361

362

363

364

365

366

367

368

369

370

371

372

373

374

375

376

377

FOXO1. Journal of Cancer 6:1295-1305. 10.7150/jca.13176

Weissmueller S, Manchado E, Saborowski M, Morris JPt, Wagenblast E, Davis CA, Moon SH, Pfister NT, Tschaharganeh DF, Kitzing T, Aust D, Markert EK, Wu J, Grimmond SM, Pilarsky C, Prives C, Biankin AV, and Lowe SW. 2014. Mutant p53 drives pancreatic cancer metastasis through cell-autonomous PDGF receptor beta signaling. Cell 157:382394. 10.1016/j.cell.2014.01.066

Wishart DS. 2008. DrugBank and its relevance to pharmacogenomics. Pharmacogenomics 9:1155-1162. 10.2217/14622416.9.8.1155

Wishart DS, Knox C, Guo AC, Shrivastava S, Hassanali M, Stothard P, Chang Z, and Woolsey J. 2006. DrugBank: a comprehensive resource for in silico drug discovery and exploration. Nucleic acids research 34:D668-D672.

Zhen DB, Rabe KG, Gallinger S, Syngal S, Schwartz AG, Goggins MG, Hruban RH, Cote ML, McWilliams RR, Roberts NJ, Cannon-Albright LA, Li D, Moyes K, Wenstrup RJ, Hartman AR, Seminara D, Klein AP, and Petersen GM. 2015. BRCA1, BRCA2, PALB2, and CDKN2A mutations in familial pancreatic cancer: a PACGENE study. Genetics in medicine : official journal of the American College of Medical Genetics 17:569-577. 10.1038/gim.2014.153

Zhuang Y, Feng Q, Ding G, Zhao M, Che R, Bai M, Bao H, Zhang A, and Huang S. 2014. Activation of ERK1/2 by NADPH oxidase-originated reactive oxygen species mediates 
Figure legends

384 Figure 1. Gene ontology (GO) enrichment analysis on the direct protein targets (DPTs) and DPTassociated genes of aspirin: (A) GO biological process (BP) analysis, (B) GO cell component (CC) analysis and (C) GO molecular function (MF) analysis.

Figure 2. The mining of genetic alterations connected with aspirin-associated genes in cancer studies with the cBio cancer genomics portal: (A) prostate cancer, (B) pancreatic cancer, $(\mathrm{C})$ small-cell lung cancer, (D) colorectal cancer, (E) bladder cancer, (F) endometrial cancer, (G) non-small-cell lung cancer, and $(\mathrm{H})$ renal cell carcinoma.

Figure 3. A visual display of the gene network connected to genes in cancer: (A) prostate cancer, 
Table $\mathbf{1}$ (on next page)

Identification of DPT-associated genes using mentha 
1 Table 1. Identification of DPT-associated genes using mentha

\begin{tabular}{lll}
\hline$\#$ & DPT of aspirin & DPT-associated genes \\
\hline 1 & PTGS1 & PTGS2, CAV2, CAV1, PTGIS, NCL \\
\hline 2 & PTGS2 & EP300, USP22, COPS7A, ELAVL1, CAV1, ELMO1, COPS5, DERL1, \\
& & NUCB1, PTGIS, CTNNB1, TP53, PTGS1, APP, CELF2, AGTR1, NOS2 \\
\hline 3 & AKR1C1 & COMMD8, TFF3, PTPN3, MAPK3 \\
\hline & PRKAA1 & RIMB3, TRIP6, L3MBTL3, ABI1, ARHGAP22, HMBOX1, NRBF2, \\
& & MTFR2, PRKAG2, VPS37B, STK11, CDC37, FKBP5, HOMEZ, RBPMS, \\
& & THAP1, PRKAB1, IKZF3, MAGED1, ROPN1, PHC2, SDE2, PNMA5, \\
& & CHERP, VPS52, CAMKK1, RFX6, INO80E, EMILIN1, THAP7, PPM1E, \\
& & WORC4, LZTS2, XRN2, PLEKHA4, MTUS2, UBXN11, CRTC2, TFPT, \\
& & CRBN, TXNIP, ABI2, HDAC5, SSX2IP, CTBP1, TOMM34, USP10, \\
& & TSC22D4, TRIM27, MAP3K7, PPM1F, FANCA, BHLHE40, ZBED1, \\
& & APRT, CFTR, ACACA, ULK1, CDX4, HSP90AB1, PRKAB2, KIF1C, \\
& & FANCG, HSP90AA1, FSBP, HSPB1, CPE, PPP2CA, NEDD1, PSMD11, \\
& & MDM4, EPM2A, TP53, MTOR, PRKAA2, AES, GOLGA2, PPM1A, TSC2 \\
\hline 5 & ERRB1, ARRB2, HDAC7, KAT5, COPS5, EDN1, SCR \\
\hline 6 & IKBKB & PRKCA, CREBBP, IRAK1, ERC1, CTNNB1, FANCA, PPP2CA, TP53,
\end{tabular}
AURKA, COPS4, MUC1, TNFAIP3, TNFRSF1A, AKT1, PRKCD, MAP3K1, MAVS, TNF, PRKCE, TRIM21, RIPK1, RICTOR, RELA, TRAF3IP2, NCOA3, COPS3, STAP2, MAP3K7, NFKB1, MAP3K14, TRAF1, TRAF2, CDC37, NFKBIB, KEAP1, NFKBIA, CHUK, IKBKG, EGLN3, COPS7A, USP18, LATS2, HOMER3, BTRC, PPM1B, BCL10, TAX, JUN, HSP90AB1, SRC, TRIM27, PEBP1, MTOR, HTT, MEOX2, CSNK2B, HNRNPU, PRKCQ, IKBKE, TWIST1, MAPK14, TRIM40, BRAP, NLRC5, RPTOR, TNIP2, PELI1, NEDD4L, TRAPPC9, TP63, CUEDC2, TAB2, KLHL21, FAF1, TRAF6, FOXO3, TP73, HSP90AA1, GLI1, PPP2R3C, CSF2RA, TSC1, COPS5, NAA20, PPP1CA, PRKDC, CSF2RB, CLTC, PPARG, PLK1, ROCK1, MAP3K11, PRKCI, ORF71, SNAP23

\begin{tabular}{lll}
\hline 7 TP53 & MT1A, CDK1, S100A6, BRCC3, RPL5, BANP, BRCA2, UFD1L, GPX2, \\
& CDKN1A, HSP90B1, P0DMV9, P0DMV8, RCC1, NQO1, XRCC1, CREB1, \\
& HNF4A, MTOR, TP53, BMI1, PPIF, NMT1, PHB, ZBTB2, SFN, YWHAZ, \\
& VRK2, SET, HECW1, Q7L7W2, BRE, RBBP6, HSPA4, UBB, TOP2A, \\
& VDR, EEF2, TPT1, HSC82, TNFAIP3, MDM2, NFKBIA, MAPK3, \\
\hline 8 HSPA5 & AMFR, PPP2R2B, MAPRE1, SIL1, ERLEC1, CFTR, Q6T424, SEC61A1, \\
& CBL, SNW1, MAP1LC3A, DMKN, AGO4, SPG20, OS9, EIF2AK3, \\
& DNAJB11, HNRNPA3, PDIA6, MTNR1A, MTNR1B, HSPA8, DNAJC10, \\
& PAWR, SH3BP4, SEC63, UBQLN4, HSPBP1, AKT1, YWHAB, CPT1A, \\
& GRB2, RELA, EP300, SQSTM1, GPX7, DPH1, HDAC6, UCHL5, \\
\hline
\end{tabular}




\begin{tabular}{|c|c|c|}
\hline & & $\begin{array}{l}\text { DNAJC1, UBL4A, UBE3A, ID2, DNAJC3, FUS, TMEM132A, VHL, CLU, } \\
\text { P01266, VIM, RPN1, AIRE, ERLIN2, TP53, RAF1, EGFR, F7VJQ1, SVIL, } \\
\text { PRNP, ERN1, HNRNPA1, FCHSD2, A2M, LDLR, HSPB1, KRT8, PIAS1, } \\
\text { P0DMV8 }\end{array}$ \\
\hline 9 & RPS6KA3 & $\begin{array}{l}\text { BARX1, EIF3C, CREBBP, CSNK2B, TRAF2, YBX1, SMS, HIST1H3J, } \\
\text { PEA15, FBXO43, MAPT, FGFR1, ATP5J, MAPK1, PDPK1, MAPK3, } \\
\text { NFKBIA, HMGN1, H2AFX, MASP1 }\end{array}$ \\
\hline 10 & NFKBIA & $\begin{array}{l}\text { MTOR, ZNF212, NFKBIB, ARRB2, UBA52, RPS6KA1, COMMD1, } \\
\text { DNAJA3, UBE2D3, PTPN13, NEDD9, CUL1, SUMO4, ARRB1, VCP, } \\
\text { REL, UL54, FBXW11, SKP1, RELB, IKBKG, BTRC, RELA, TBK1, } \\
\text { G3BP2, HIF1AN, RWDD3, PIK3R1, CSNK1A1, UBE2D1, COPS2, UBE2I, } \\
\text { CSNK2B, MAPK14, MAP3K14, BARD1, IKZF4, NCOR2, POM121, } \\
\text { UBE2D2, DYNLL1, UBE2L3, NFKB2, MAP3K1, ITPK1, IKBKE, } \\
\text { RPS6KA3, CDC34, ABL1, TP53, RPS3, ATF4, UBC, IKBKB, CHUK, } \\
\text { NFKB1, POLRMT, SOCS3, MAP3K7, PRKCA, PSMA4, PIR, AURKA, } \\
\text { IKBKAP, BCL10, CAPN1, HOXB7, HNRNPA1, SLC25A4, PSMA2, } \\
\text { LYL1, TNF }\end{array}$ \\
\hline 11 & NFKB2 & $\begin{array}{l}\text { NFKB2, REL, TSC22D3, FBXW7, FBXW11, RELA, RELB, BTRC, } \\
\text { MAP3K14, DPF2, EPS8, NFKBIB, STAT3, BCL3, NFKB1, CHUK, } \\
\text { MAP3K8, MEN1, NKRF, NR3C1, SP1, P0C722, P0C723, P03207, } \\
\text { NFKBIE, NFKBIA }\end{array}$ \\
\hline 12 & CRP & $\begin{array}{l}\text { RPL13A, DGCR14, MAPK3, LAMC3, CRP, APCS, C1QA, CFH, MAPK1, } \\
\text { FCGR2C, HIST1H1A, HIST2H2AC, GMPPA, FN1, SPA, FCN2, GLUD1 }\end{array}$ \\
\hline 13 & SELP & $\begin{array}{l}\text { SELP, CD24, COL18A1, SELPLG, SNX17, SNX27, AP1M1, VCAN, } \\
\text { GP1BA, EZR }\end{array}$ \\
\hline 14 & TBXA2R & $\begin{array}{l}\text { GNA11, GNB1, KCNMA1, PSME3, NME2, AAMP, YWHAZ, GNB2L1, } \\
\text { RPGRIP1L, PKN1, RAB11A, PRDX4, GNAQ, GHRL, GNG2, GNAS, } \\
\text { KCNMB1, GPRASP1, WDR36, GRK5, PRKG1, GNA13, GRK6, PTGIR, } \\
\text { PRKCA, PSMA7, SIVA1, PRKACA, GNAI2 }\end{array}$ \\
\hline 15 & REN & AGT, ATP6AP2 \\
\hline 16 & MMP9 & $\begin{array}{l}\text { COL18A1, CXCL5, CXCL6, VCAN, COL1A2, CLU, TIMP1, TGFB1, } \\
\text { SRGN }\end{array}$ \\
\hline 17 & NOS3 & $\begin{array}{l}\text { ACTN2, CTNNB1, MPRIP, IMMT, EFEMP1, H3F3B, TXNDC11, HTRA1, } \\
\text { RNF31, FIS1, CAV1, NOSTRIN, AKT1, NOSIP, ST13, GCDH, ACTB, } \\
\text { GUCY1B3, GOLGA2, CDC37, MAGEA11, ACTN4, EFEMP2, UMPS, } \\
\text { P0DP23, P0DP24, P0DP25, HSP90AA1, TAX, APOE, P0DP30, P0DP31, } \\
\text { P0DP29 }\end{array}$ \\
\hline 18 & IL10 & IL10RA, IL10RB, A2M \\
\hline
\end{tabular}


Table 2 (on next page)

KEGG pathway associated with cancer 
1 Table 2. KEGG pathway associated with cancer

\begin{tabular}{|c|c|c|c|}
\hline KEGG pathway-Term & Count & $p$ Value & Genes \\
\hline $\begin{array}{l}\text { bta05200: Pathways in } \\
\text { cancer }\end{array}$ & 78 & $2.79 \mathrm{E}-20$ & $\begin{array}{l}\text { GNA13, HSP90AB1, PTGS2, MMP9, } \\
\text { GNA11, PPARG, NFKB1, NFKB2, PTEN, } \\
\text { TGFB1, CTNNB1, GLI1, AKT1, EDNRA, } \\
\text { AGTR1, CDKN2A, CASP8, PRKACA, } \\
\text { GNG2, NOS2, CHUK, PRKCA, CTBP1, } \\
\text { HSP90AA1, BCR, ROCK1, RELA, TP53, } \\
\text { RB1, DAPK3, CDK2, RAD51, DAPK1, } \\
\text { MAPK1, HIF1A, GNAQ, GNB1, LAMC3, } \\
\text { JUN, MAPK3, MAPK9, MDM2, PIAS2, } \\
\text { GNAS, MAPK8, TRAF1, TRAF2, FGFR1, } \\
\text { GNAI2, PML, EGLN3, NFKBIA, BCL2L1, } \\
\text { PTK2, BCL2, TRAF6, PIK3R1, FN1, } \\
\text { AXIN1, DVL2, MSH2, VHL, CREBBP, } \\
\text { BRCA2, SMAD3, SMAD2, STAT3, } \\
\text { HSP90B1, LAMA4, CDKN1A, HDAC2, } \\
\text { HDAC1, GSK3B, IKBKG, PLCG2, MTOR, } \\
\text { IKBKB, ABL1 }\end{array}$ \\
\hline $\begin{array}{l}\text { bta05215:Prostate } \\
\text { cancer }\end{array}$ & 28 & $6.53 \mathrm{E}-13$ & $\begin{array}{l}\text { HSP90AB1, FGFR1, NFKBIA, NFKB1, } \\
\text { PTEN, CTNNB1, AKT1, PDPK1, BCL2, } \\
\text { CHUK, PIK3R1, HSP90AA1, RELA, } \\
\text { CREB1, CREBBP, TP53, RB1, CDK2, } \\
\text { MAPK1, CDKN1A, ATF4, HSP90B1, } \\
\text { GSK3B, MAPK3, IKBKG, MDM2, MTOR, } \\
\text { IKBKB }\end{array}$ \\
\hline $\begin{array}{l}\text { bta05212:Pancreatic } \\
\text { cancer }\end{array}$ & 21 & $1.02 \mathrm{E}-09$ & $\begin{array}{l}\text { RELA, TP53, SMAD3, BRCA2, SMAD2, } \\
\text { NFKB1, BCL2L1, RB1, STAT3, TGFB1, } \\
\text { RAD51, AKT1, MAPK1, CDKN2A, } \\
\text { MAPK3, IKBKG, MAPK9, MAPK8, } \\
\text { IKBKB, CHUK, PIK3R1 }\end{array}$ \\
\hline $\begin{array}{l}\text { bta05222:Small cell } \\
\text { lung cancer }\end{array}$ & 24 & $2.04 \mathrm{E}-09$ & $\begin{array}{l}\text { TRAF1, TRAF2, PTGS2, RELA, TP53, } \\
\text { NFKBIA, NFKB1, BCL2L1, RB1, PTEN, } \\
\text { CDK2, AKT1, LAMA4, PTK2, LAMC3, } \\
\text { BCL2, IKBKG, PIAS2, NOS2, TRAF6, } \\
\text { IKBKB, CHUK, PIK3R1, FN1 }\end{array}$ \\
\hline $\begin{array}{l}\text { bta05210:Colorectal } \\
\text { cancer }\end{array}$ & 16 & 8.94E-06 & $\begin{array}{l}\text { MSH2, TP53, SMAD3, SMAD2, TGFB1, } \\
\text { CTNNB1, AKT1, MAPK1, GSK3B, JUN, } \\
\text { BCL2, MAPK3, MAPK9, MAPK8, PIK3R1, } \\
\text { AXIN1 }\end{array}$ \\
\hline
\end{tabular}




\begin{tabular}{|c|c|c|c|}
\hline $\begin{array}{l}\text { bta05219:Bladder } \\
\text { cancer }\end{array}$ & 11 & $1.21 \mathrm{E}-04$ & $\begin{array}{l}\text { MAPK1, CDKN1A, CDKN2A, MMP9, } \\
\text { MAPK3, TP53, MDM2, RB1, DAPK3, SRC, } \\
\text { DAPK1 }\end{array}$ \\
\hline $\begin{array}{l}\text { bta05213:Endometrial } \\
\text { cancer }\end{array}$ & 11 & $9.87 \mathrm{E}-04$ & $\begin{array}{l}\text { AKT1, MAPK1, PDPK1, GSK3B, MAPK3, } \\
\text { TP53, FOXO3, PTEN, PIK3R1, AXIN1, } \\
\text { CTNNB1 }\end{array}$ \\
\hline $\begin{array}{l}\text { bta05223:Non-small } \\
\text { cell lung cancer }\end{array}$ & 11 & 0.002081 & $\begin{array}{l}\text { AKT1, PRKCA, MAPK1, PDPK1, } \\
\text { CDKN2A, MAPK3, PLCG2, TP53, RB1, } \\
\text { FOXO3, PIK3R1 }\end{array}$ \\
\hline $\begin{array}{l}\text { bta05211:Renal cell } \\
\text { carcinoma }\end{array}$ & 11 & 0.007081 & $\begin{array}{l}\text { AKT1, MAPK1, HIF1A, VHL, JUN, } \\
\text { MAPK3, CREBBP, EGLN3, RAP1B, } \\
\text { TGFB1, PIK3R1 }\end{array}$ \\
\hline
\end{tabular}

2 
Figure 1

Gene ontology (GO) enrichment analysis on the direct protein targets (DPTs) and DPTassociated genes of aspirin

A GO biological process (BP) analysis, B GO cell component (CC) analysis, C GO molecular function (MF) analysis. 


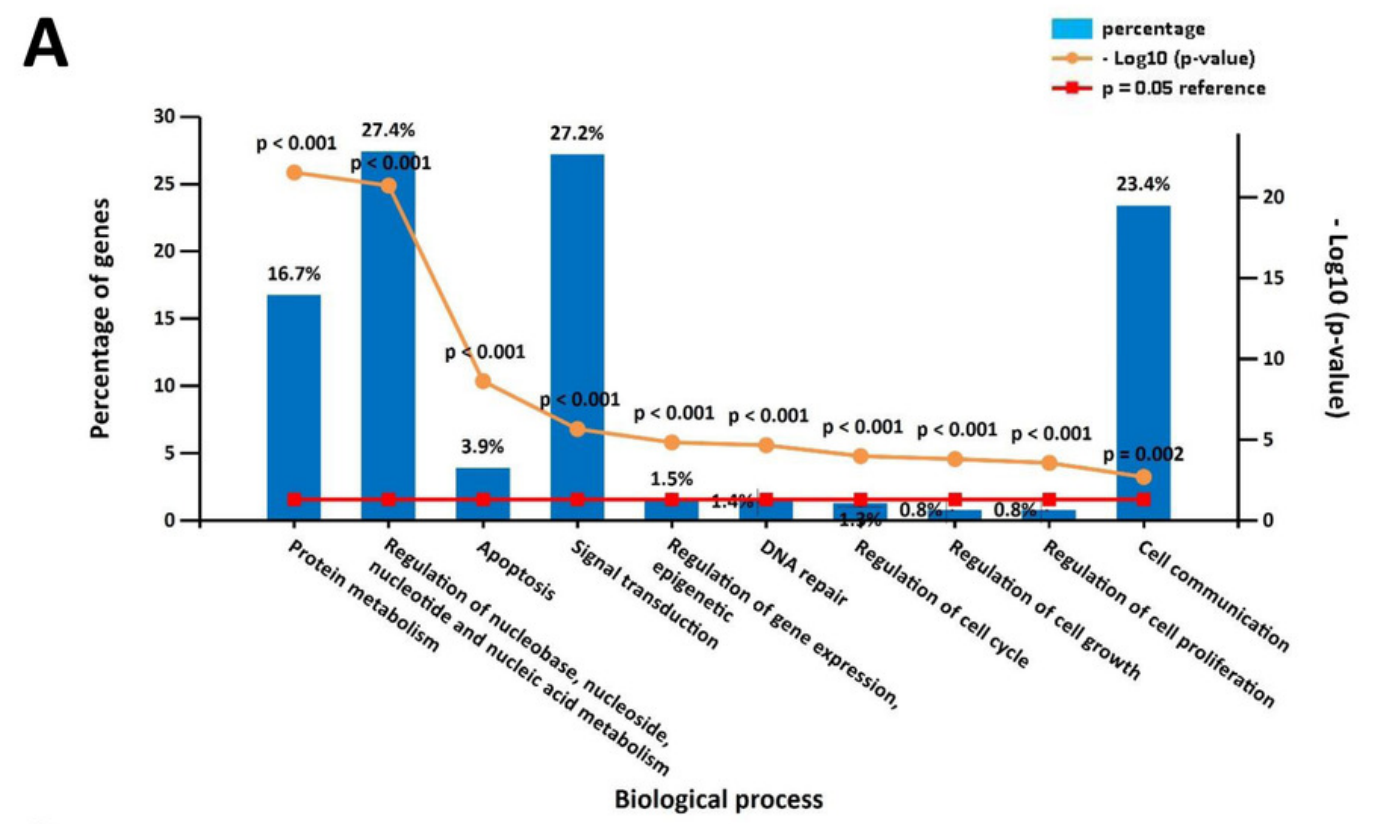

B

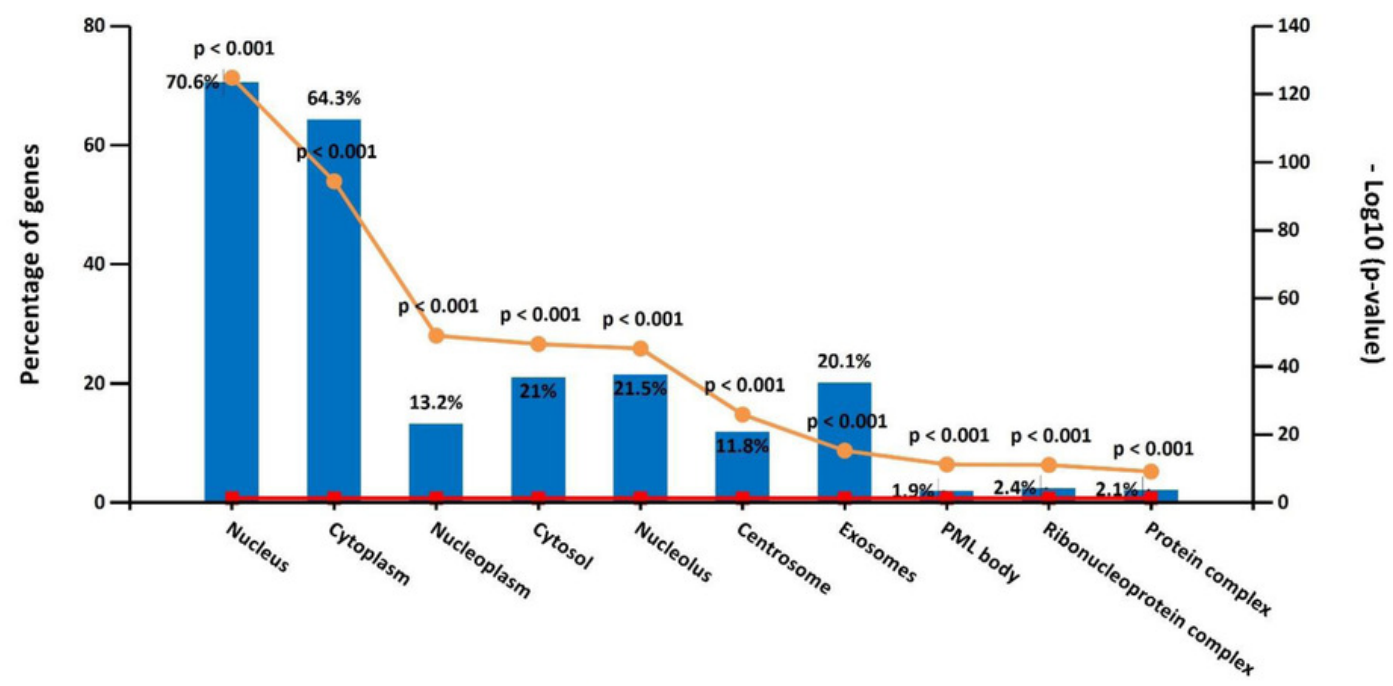

C

Cellular component

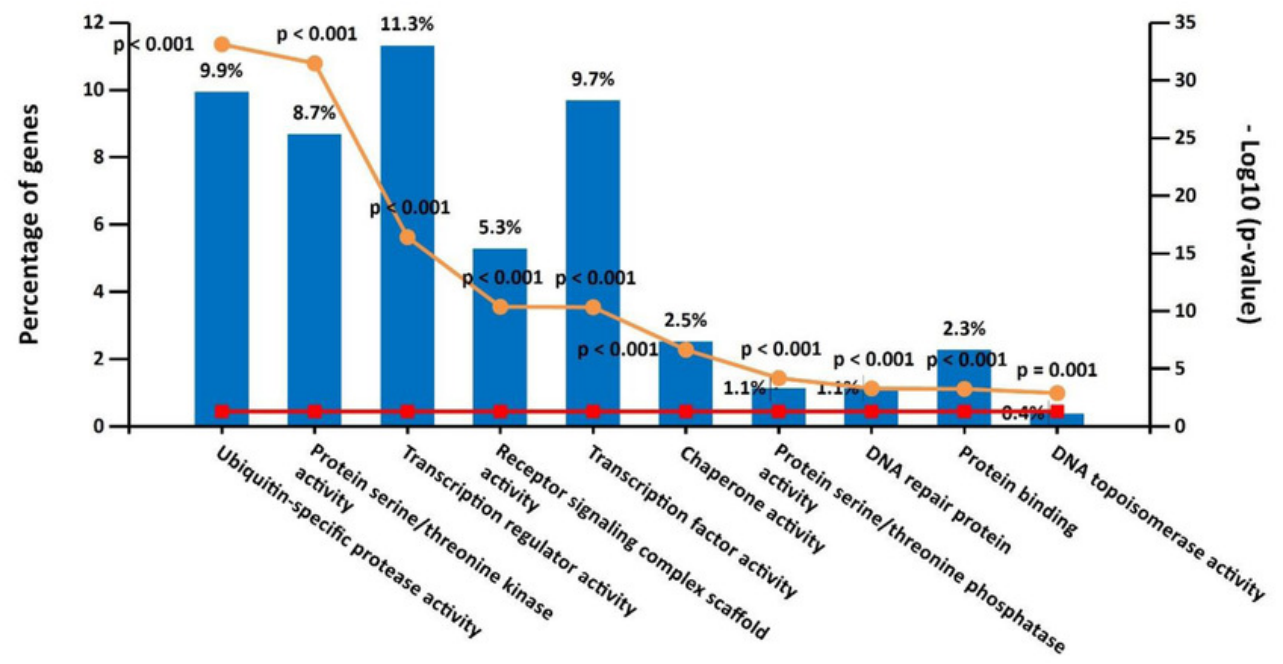


Figure 2

Mining genetic alterations connected with aspirin-associated genes in cancer studies with the cBio cancer genomics portal

A Prostate cancer, B Pancreatic cancer, C Small-cell lung cancer, D Colorectal cancer, E Bladder cancer, F Endometrial cancer, G Non-small-cell lung cancer, H Renal cell carcinoma. 
A

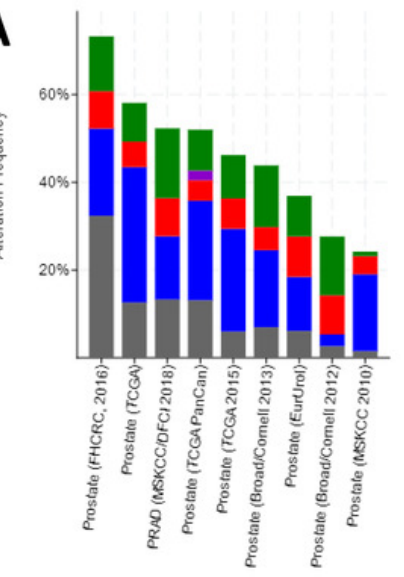

C

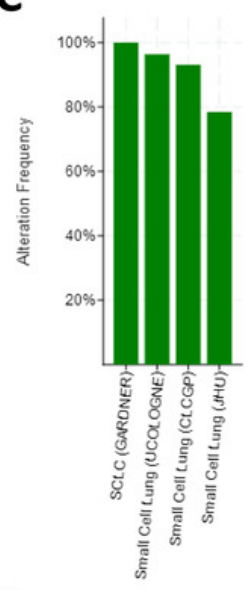

E
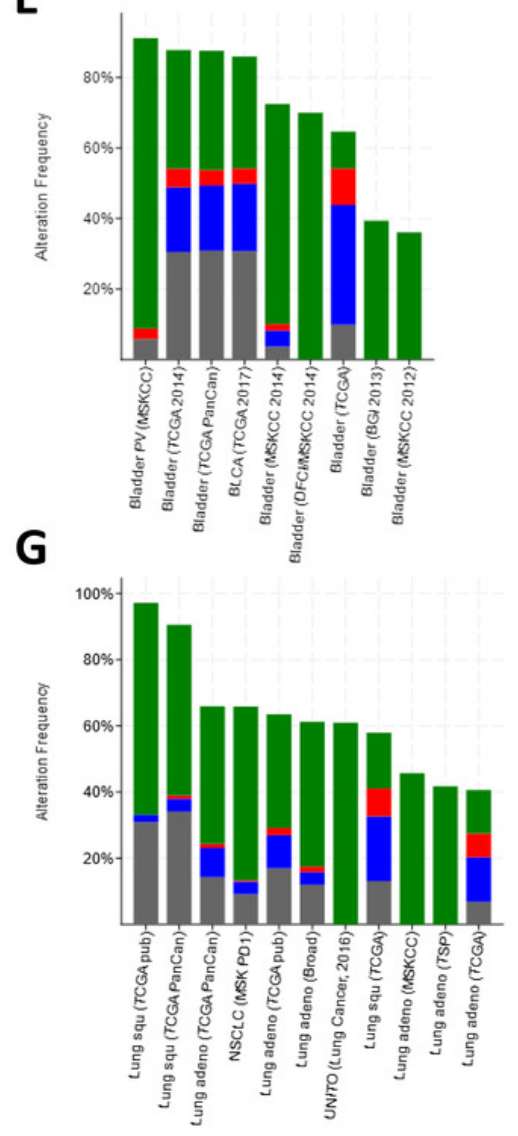

B

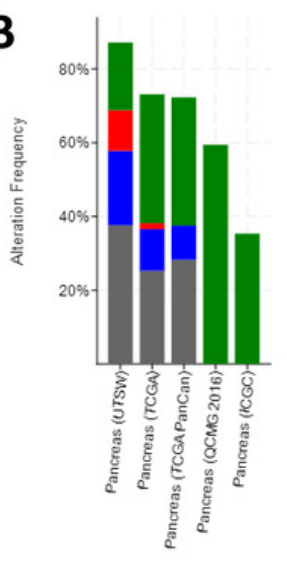

D

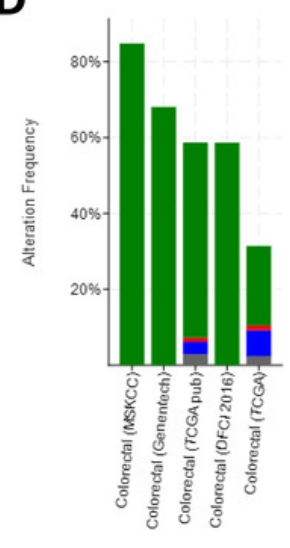

$\mathbf{F}$

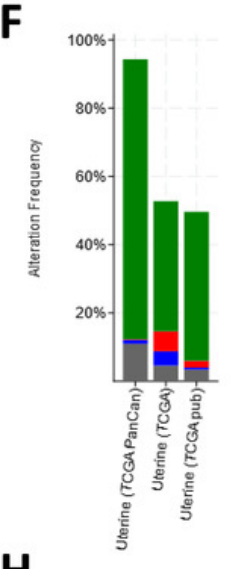

H

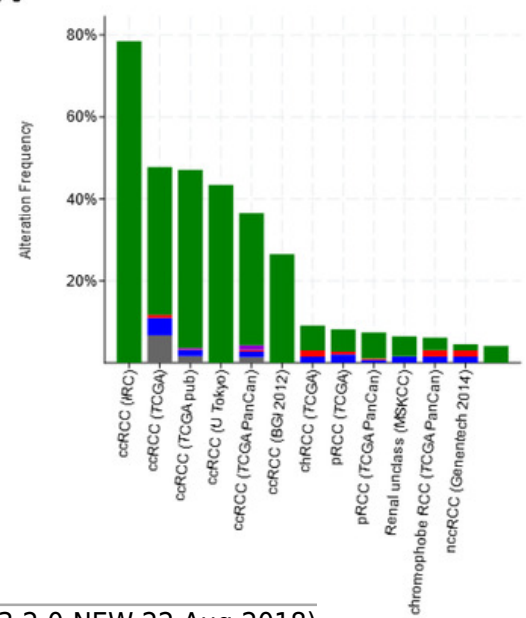

Peer] reviewing PDF | (2018:04:27803:2:0:NEW 22 Aug 2018) 


\section{Figure 3}

Visual display of the gene network connected to genes in cancer

A Prostate cancer, B Pancreatic cancer, C Small-cell lung cancer, D Colorectal cancer, E Bladder cancer, F Endometrial cancer, G Non-small-cell lung cancer, H Renal cell carcinoma. 
A

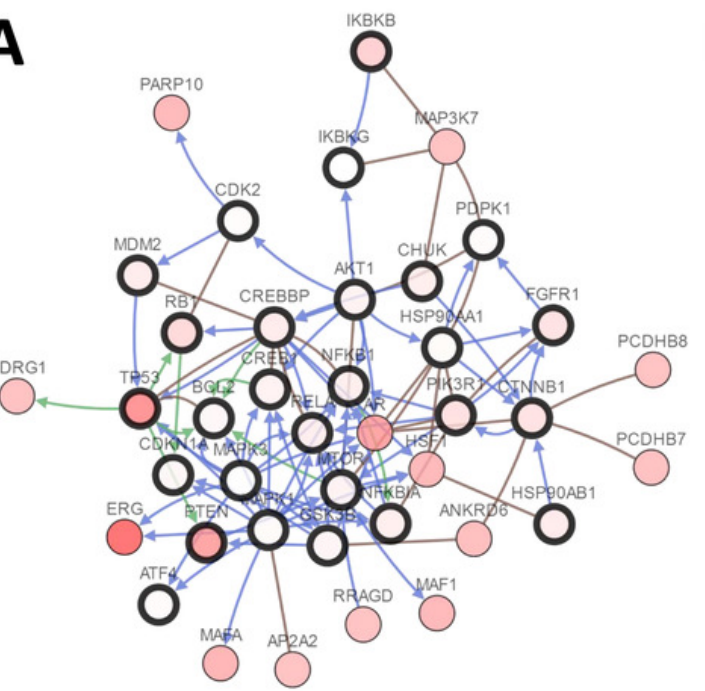

C

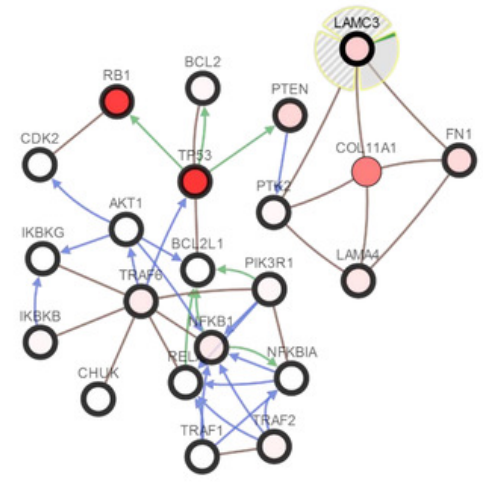

E

E

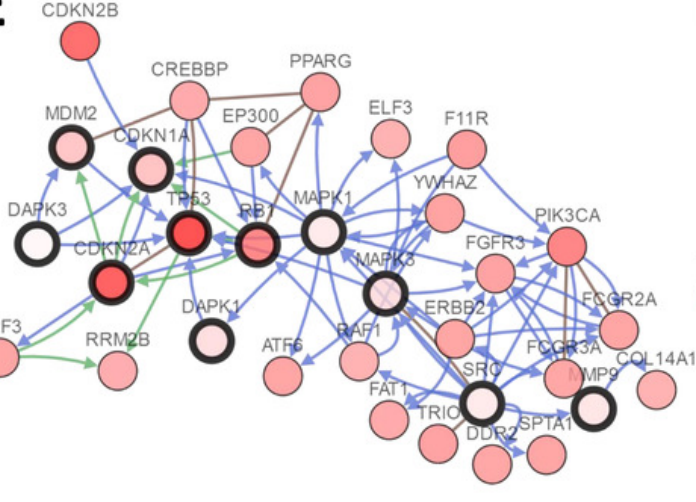

B

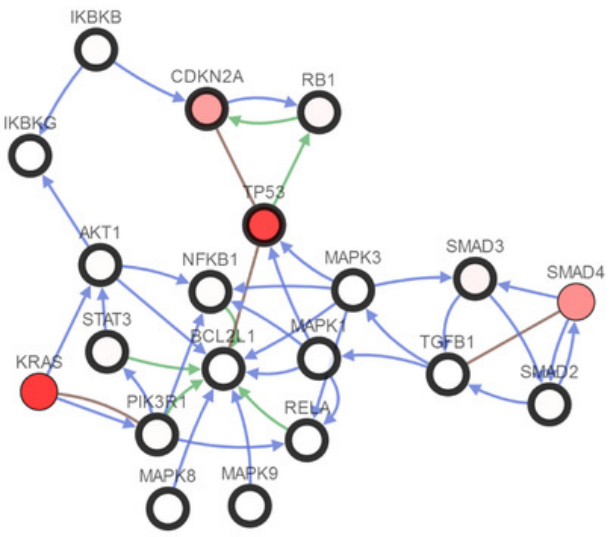

D
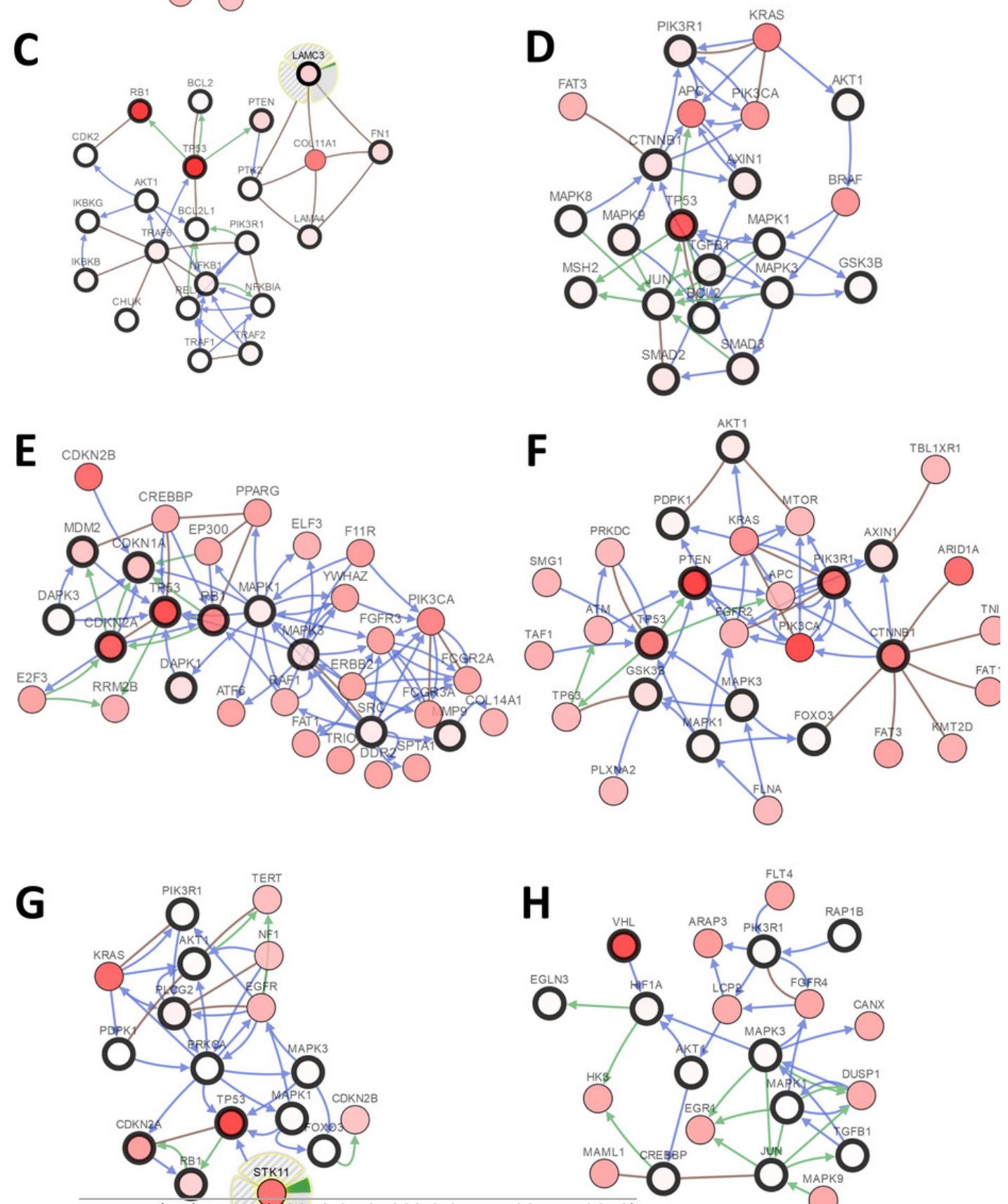

PeerJ reviewing PDFY(2018:04:27803:2:0:NEW 22 Aug 2018) 